\title{
ARE THERE SUCH THINGS AS THEORIES OF TRAINING EQUALLY COMPARABLE TO THEORIES OF LEARNING? LITERATURE REVIEW
}

\author{
Fuad Cholisi
}

\begin{abstract}
This article provides a literature review on the comparison between theories of learning and theories of training. Despite their equally vital roles in the improvement of human capital quality, it turns out that almost all of the training theories have been based, as their ground, on the learning theories. The learning theories, on the other hand, have long been established with a firm ground philosophically, psychologically, and pedagogically. This strong foundation has made learning theories such a trade mark that looks so firm, stable and reliable. As a general finding, this article concludes that there are indeed some theories of training that are applicable for individual and organizational training. However, such theories are obviously not equally comparable to the theories of learning.
\end{abstract}

Key words: the learning theories, the training theories, individual learning, organizational learning.

Learning and training are two household terms in the areas of Human Resource Management and Organizational Behaviour, partly because they are by and large considered as two essential elements for striving for excellence. Training and employee development, which involve learning activities at both individual and organizational level, are regarded as a vital component in maintaining organizations' competitiveness. In Human Resource Management and Human Resource Development context, these two elements play a very significant role in that they are part of the key means by which the inefficiencies of the emplt oyment relationship can be reduced, and which will lead to the attainment of competitive labour market outcomes (Garavan, 1997). According Garavan, the most common organizational outcomes which can be achieved include quality, employee empowerment, teamwork and multi-skilling.

The recent years have seen a growing interest by organizations in learning and training, and much has been written regarding these compelling topics. Yet, their meaning and concepts have so far remained a vast area of debate. While 
psychological theories of learning have been established as a considerably robust foundation of the area of study, despite the wide variety of approaches proposed by different experts and different theoretical schools, dissatisfaction with the theoretical foundation of training has been expressed by various authors (see Al-Khayyat and Elgamal, 1997). With regard to training, Al-Khayat and Elgamal are particularly concerned about the fact that the literature is still generally non-empirical and nontheoretical. The question arising at this point is: if it is true that we have theories of learning, do we also have theories of training? This essay aims to address the question above by presenting a brief overview of the theories of learning, and a discussion about the existing theories related to training, before finally attempting to seek the link, as well as to draw a line between the two in order to answer the question.

\section{Theories of Learning: A Brief Overview}

The discussion about learning theories cannot be separated from major theoretical approaches in psychology. This section will deal with a brief overview of the key concepts of learning within psychological science. As was stated in the previous section, learning has been defined in different ways, and defining learning can prove complicated due to the multiple uses of the term. Smith (1982) concluded that the existing definitions imply the term learning is used to refer to: (1) the acquisition and mastery of what is already known about something (described as product), (2) the extension and clarification of meaning of one's experience (described as function), or (3) an organized, intentional process of testing ideas relevant to problems (described as a process).

\section{The Behaviourist Approach to Learning}

The basic assumption of the behaviourist approach is the idea of association or associative learning (Stewart, 1999). The behaviourists are not interested in exploring what processes go on inside the organism; rather they focus their attention 
on the study of behaviour and the actions which can be observed, measured, and controlled. Learning is regarded as a matter of stimulus-response. The behaviourist approach draws on the insights derived from classical conditioning, i.e., when the learner is conditioned to give the same response to a different stimulus, and operant conditioning, i.e., if the learner is conditioned to give a different response to the same stimulus (Cotton, 1998).

The key figure associated with classical conditioning is Pavlov (Patric, 1992; McKenna, 1994; Mullins, 1999; Cotton, 1998; Stewart, 1999), who through his experiments with the digestive system of dogs, identified the basic principle of classical conditioning, where conditioned response emerges not only as a result of unconditioned stimulus, but also in relation with conditioned stimulus, and learning can take place as a result of the association between unconditioned stimulus and unconditioned response. In brief, classical conditioning is about a pattern of behaviour which results from an association of one event with another.

Operant conditioning is generally recognized as Skinner's thesis, although the idea basically came from Thorndike, who gave much thought to the relationship between stimulus and response. Having observed the consistent behaviour of cats escaping from puzzle boxes by trial and error, he concluded that learning was a result of the gradual strengthening of connections between a stimulus and a response. Thorndike proposed three laws to explain the learning of animals and human beings: the law of readiness, the law of exercise, and the law of effect, but the most influential to the operant conditioning theory is the last one. The law of effect is concerned with the strengthening or weakening of a connection as a result of its consequences, i.e., satisfying or annoying state of affairs.

Skinner elaborated and advanced Thorndike's law of effect in his thesis of operant conditioning (Patrick, 1992; McKenna, 1994; Knowles et al, 1998; Mullins, 1999). Operant conditioning differs from classical conditioning in that in operant conditioning, some response has to be made by the learner before his or her behaviour is reinforced (McKenna, 1994). From his experiment with a rat in a box with a lever which could be depressed to activate a mechanism to deliver a food pellet, Skinner gained enough evidence to strengthen the Stimulus-Response 
connection. He also found that the behaviour was more resistant to extinction, i.e., if reinforcement is expected but is not forthcoming, the response associated with it could become extinguished. In operant conditioning, it is possible to control and change behaviour by reinforcing in a systematic way the desirable actions. This technique, which can be used to speed up certain processes of learning is called shaping, which works by selecting an appropriate reinforcer to suit the occasion in order to move the individual to the desired behaviour.

\section{Cognitive Learning}

The contrast between a behaviourist approach to learning and a cognitive approach is that the former conceptualised the trainee as being passive and that learning was largely an automatic process. In cognitive approach, on the other hand, the learner was conceptualized as being active, not in the sense of performing, but by bringing to bear different strategies, perspectives and interpretations on the subject matter in seeking a coherent understanding. Secondly, in cognitive learning there is a change in what the learner knows rather than what he or she does. The processing of knowledge is therefore important (Patrick, 1992; McKenna, 1994).

Cognitive theory of learning was pioneered by psychologists such as Kohler, Tolman, Piaget, and Gagne. For them, learning is viewed as a form of information processing through several stages. Gagne's theory of learning, in particular, is a simple one, where learning was perceived as an individual activity in accessing, processing and transforming information from their physical and social environment (Stewart, 1999; Mullins, 1999). This theory was the first to explain the role of cognition and cognitive processes in individual learning, which was later on elaborated by its subsequent proponents to support the view that learning and behaviour can be separate in time. During the learning process, individuals formulate cognitive maps of their experience and environments, which are applied to inform and shape behaviour independently from the immediate context of the behaviour itself (Stewart, 1999). In the later development of the theory, the concept of cognitive map has been related to the concept of 'schema', an internal structure 
developed through experience that organises incoming information in relation to previous experience.

An influential approach which is based on cognitive theory is experiential learning, which emphasises the cyclical nature of learning and its active nature. The central figure behind this theory of learning is David Kolb, who developed a fourstage model of learning by doing which he called Experiential Cycle or Learning Cycle. In this model, learning is described as a cycle consisting of four stages of learning activities: concrete experiences stage, observational and reflective stage, abstract conceptualisation stage, and active experimentation stage.

Mullins (1999) concluded that this model provides useful insights into the nature of learning for the following reasons:

- It demonstrates that there is no end to learning but only another turn of the cycle

- Learners are not passive recipients but need to actively explore and test the environment

- It identifies the importance of reflection and internalisation

- It is a useful way of identifying problems in the learning process.

Kolb's learning cycle was later on simplified and refined by Honey and Mumford into a learning style model which identifies four types of learners: the activist, the reflector, the theorist, and the pragmatist (see Woodall and Winstanley, 1998).

\section{Andragogy}

Another development in personal psychology and learning which has also been instrumental is the work of Malcolm Knowles on the principles of adult learning. He calls his theory 'andragogy' in opposition to 'pedagogy', which is the science of teaching children. Knowles rejects theories of teaching which are based upon behaviourist principles, cognitive theory, and motivation and personality theory. He argues that these theories were based on studies of animals and children - 
not adults. According to Knowles, adult learning should be based on the following distinctive principles of andragogy:

- The need to know

- The learner's self-concept

- The role of learners' experiences

- Readiness to learn

- Orientation to learning

- Motivation (Knowles, et al, 1998).

Knowles's principles of andragogy have had a profound influence on approaches to employment development, since the learning-styles preferences suggested by the theory can be maximised by organizations to improve their training and development programmes.

\section{Training: What the Literature Holds}

As with the case of learning, it is not easy to find a comprehensive definition of training which is at the same time appropriate for particular contexts due to its overlap with other areas in applied psychology (Stammers, 1975). Most of the existing definitions give the meaning of training in terms of organizational and jobrelated context. A quite popular definition of training, however, is given by the Department of Employment's Glossary of Taining Terms, as cited by Stammers and Patrick (1975), as well as by Patrick (1992) as "The systematic development of the attitudes/knowledge/skill behaviour pattern required by an individual in order to perform adequately a given task or job..."

Training is closely related to the theories and principles of learning, and as far as learning is concerned, training has the following characteristics:

- It is concerned with people learning to perform mainly specific and prescribed task. 
- It adopts a normative approach by organizing the general conditions necessary for learning which enable trainees to perform the same task to the same criterion, taking into consideration of individual differences.

- It is distinct from education in that it has a more specific objectives which is to improve performance at a particular task, while education has a more wide-ranging aim and tends to be only broadly applicable to learners (Stammers, 1975; Patrick, 1992; Garavan, 1997).

In relation with setting up a model of training in organizations, Hinrichs (1976) views learning as the alteration of behaviour in its broadest sense. As he claims, the term behaviour includes any aspects of human activity, cognition, or feeling, and when becomes learned can be considered as a skill. No wonder that the literature has included skill acquisition theories as an important part of training (Stammers, 1975; Hinrichs, 1976; Patrick, 1992; Mckenna, 1994), regardless of the controversy whether such a topic should be embraced under the label of theory of training or theory of learning. The following section will be particularly concerned with this topic.

\section{Theories of Skill Acquisition: A Psychological Perspective}

Goldstein (1986) defines employee training as "the systematic acquisition of skills, rules, concepts, or attitudes that result in improved performance on the job." The definition implies that skill acquisition is one of the main issues in training. This is in compliance with what Patrick (1992) points out regarding the main goal of training. As he states, the main goal of training is concerned with the acquisition, transfer and retention of skills and knowledge. Indeed, skill acquisition is one of the main issues in training, although, as is suggested by Patrick, theories of skill acquisition focus on the processes of learning which involve qualitative changes with practice.

Various theories of skill acquisition have been proposed and developed by different experts, each of which has a particular scope. Below is a selection of skill acquisition theories summarized from the literature. 


\section{a. Crossman's Selection Theory}

From studies of repetitive manual skills which emphasized on speed, Crossman observed that cycle time decreased with practice. His observation showed that the experienced worker performed faster than the trainee. In brief, Crossman concludes that practice results in a selective effect on the operator's behaviour to the benefit of those patterns of action which are quickest, sacrificing others. In Crossman's theory, skill acquisition is viewed as a process of the human operator selecting the most appropriate method from his repertoire. In training, therefore, such repertoire may provide some hints of what the best method is to be selected by the trainer, which could be most effectively attained under the guidance through the correct patterns of action. The acquisition of speed will expectedly be encouraged by the extrinsic feedback to the trainee upon the establishment of the appropriate pattern (Stammers and Patrick, 1975).

\section{b. Fitts' Three-Phase Theory}

Fitts developed his theory from his experiments and the opinions of pilot trainers and sport coaches concerning various types of skill learning by the trainees (Stammer and Patrick (1975). According to Fitt's theory, skill develops through three phases: the cognitive, associative, and autonomous phases. These phases are characterized by overlaps and continuous transition from one to the next phase, except between the first and the third phases.

The cognitive phase deals with the acquisition of some complex task which involves an understanding of new rules and concepts before they can be efficiently executed. This is called the initial "intellectualization" process, in which both the trainer and the trainee attempt to verbalize what has to be learned. The tasks to be performed generally vary in complexity, but, unfortunately, Fitts' formulation does not allow any prediction of the degree of importance of any phase.

The associative phase is concerned with the establishment of the correct patterns of behaviour by practice, marked by gradual elimination of errors. 
Compared to the cognitive phase, this phase generally lasts longer due to the complex process to establish such "associations".

The autonomous phase of skill acquisition is characterized by two main features which, as cited by Stammer and Patrick (1975) from Fitts, consist of:

(1) gradually increasing speed of performance in tasks where it is important to improve time or accuracy scores far beyond the point where errors, as ordinarily defined, can detected, and

(2) gradually increasing resistance to stress and to interference from other activities that may be performed concurrently.

Patrick (1992) points out that during this autonomous phase skills become more automatic, and the trainee relies less and less on verbal mediation of the skill. Performance in some tasks has also shown the decreasing reliance on visual feedback, and the person has extra capacity to perform other tasks simultaneously due to redundancy of information sources. As skill progresses, more and more automation of behaviour could presumably become programmed.

\section{c. Adam's Close-Loop Theory of Motor Learning}

Adam developed a theory called the close-loop theory, and he considered the learning of graded movements as the basis of skill acquisition (Stammers and Patrick, 1975; Patrick, 1992). According to the theory, learning can be explained in terms of two constructs: a perceptual trace and a memory trace. A perceptual trace is the construct which fundamentally determines the extent of movement and is adjusted on successive repetitions on the basis of the response-produced feedback and any extrinsic feedback. A matching process between the on-going feedback of the current movement and the perceptual trace controls the execution of a movement. Prior to the use of the perceptual case, a memory trace is required to select and initiate the movement.

The close-loop theory also postulates that learning proceeds through two stages, verbal motor and then motor. As explained by Patrick (1992), the verbal 
motor stage includes both the cognitive and associative phases proposed by Fitts. In this stage extrinsic feedback is of particular importance due to its role in gradually developing a more accurate perceptual trace of the required movement. Verbal information, which is important in terms of general instructions concerning the task and feedback about previous movement, is critical in the first verbal motor stage of learning but becomes redundant in the second motor stage when the perceptual trace is well established (Stammers and Patrick, 1975). Adam's theory emphasizes the potency of feedback as a learning variable, and this has been supported by the literature. However, the theory is subject to criticisms. On the first place, it does not explain how learning can occur without the appropriate perceptual trace being developed by experiencing the correct movement. Secondly, the theory is vague about how a person can generate a new and unfamiliar movement without a corresponding perceptual trace to guide its execution (Patrick, 1992).

\section{d. Schmidt's Schema Theory of Motor Learning}

Schmidt developed a theory referred to as the schema theory of motor learning in an attempt to overcome the problems related to Adam's theory discussed above. He utilized the concept of schema previously introduced by Bartlett (Patrick, 1992). A schema functions in organising experiences resulting from the environment and in guiding future action. In his theory, Schmidt suggested the development of a motor response schema, which explains how a vast repertoire of different movement can be recalled, as well as how it is possible to generate new ones. This motor schema is made up of four dimensions of movement: the initial condition, response specification, sensory consequences, and response outcome. According to the theory, the relationship between these dimensions becomes stronger with successive repetitions of the same movement type.

Figure 2 describes how the relationship between the four dimensions works. As can be seen from the figure, the theory distinguishes between the schema required for recall and the schema needed for recognition. While the recall schema deals with response specification, the recognition schema is responsible for sensory consequences. Despite their being separate, however, these schemata are associated 
with each other, and they share initial conditions and past outcomes. The theory postulates that learning occurs as a result of feeding back error information to both schemata. As explained by Schmidt, error information can either emerges from the performer's subjective reinforcement or from extrinsic feedback given by the trainer, coach etc. (Patrick, 1992).

In brief, Schmidt's theory deals with the generation process of novel movements, where the accuracy of the relationships between aspects of a movement is considered to be determined by the amount of practice and the extent of quality of the error information.

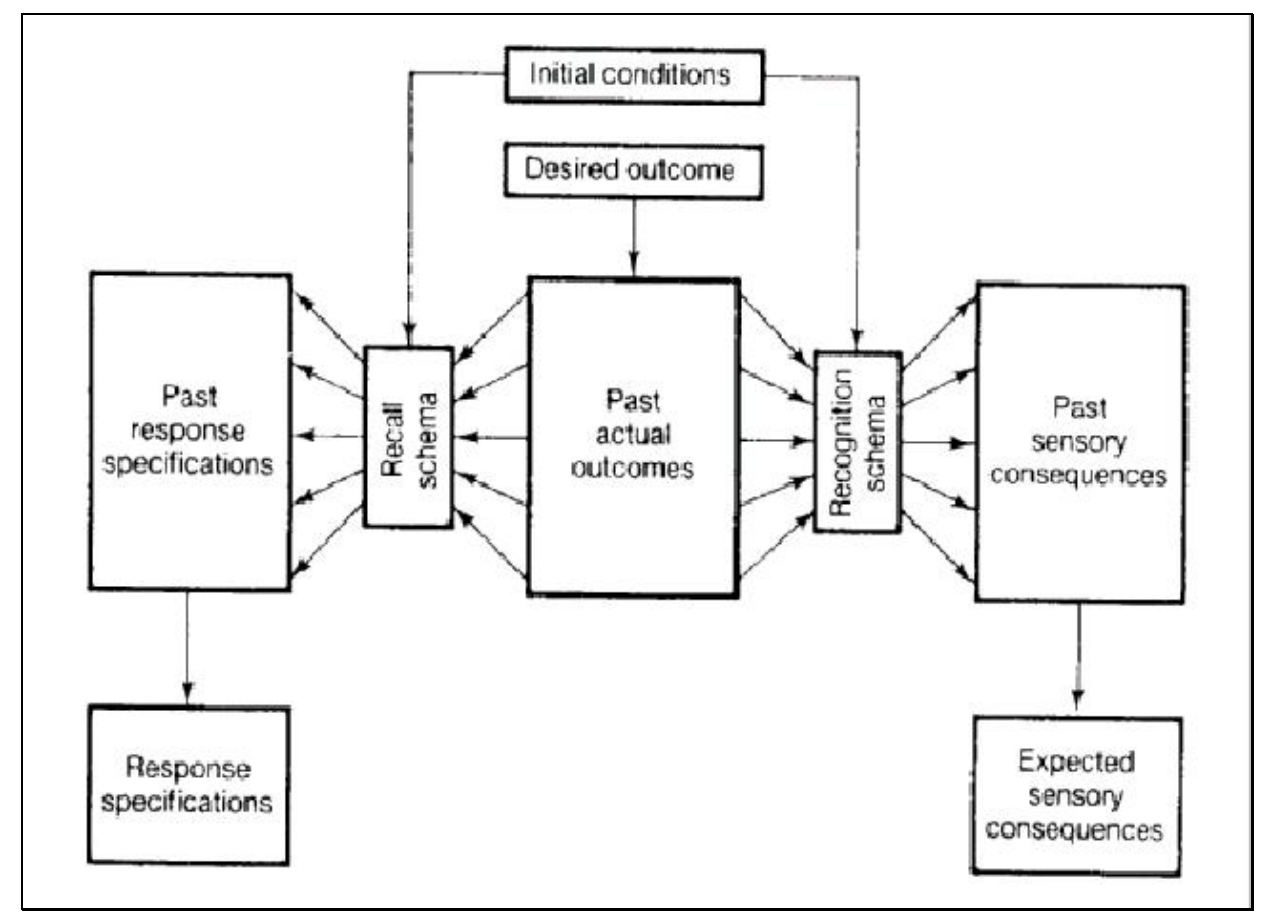

Figure 2: Schmidt's schema theory of motor learning (source: Patrick, 1992).

\section{e. Anderson's Theory of Cognitive Skill Acquisition}

Anderson suggested the stages of skill acquisition similar as those in Fitts' theory, but his hypothesis regarding the processes of the transition from the novice to expert is different from those suggested by Fitts. According to Anderson, the cognitive basis of skills plays an important role in providing a framework for 
understanding a range of skills, and skill acquisition is a process of developing and refining such rules in the transition from declarative knowledge, which is factual and can be made explicit, to a highly tuned procedural knowledge, which is implicit and not easily verbalized.

As proposed by Anderson, the development of skill involves three stages: the declarative stage, the knowledge and compilation stage, and the tuning stage. In the declarative stage, a trainee attempts to perform a new task by using some facts concerning the task in conjunction with general problem solving procedures, where working memory is heavily demanded and analogies can be useful. In the knowledge compilation stage, the trainee is developing a specific procedure from the declarative knowledge. This involves two important processes: composition, where adjacent rules are either collapsed or merged into a single more direct rule, and proceduralization, which enables a rule to incorporate more task relevant information. The tuning stage is concerned with the adjustment and improvement of the skill by means of a generalization process, a discrimination process, or a strengthening process (see Patrick, 1992).

Anderson's theory implies the importance of identifying the rule structure underlying skill performance in training in order to minimize the difficulty of constructing task-specific procedures. In his account, skill acquisition deals with the development and refinement of rules, whose constructions are achieved by doing the skill. Therefore, training must provide an opportunity for performing the task.

\section{The Systematic Model of Training}

While training can be viewed as a system which interacts with other systems such as personal selection and ergonomics, the development of training can itself be viewed as a system and can be analysed into its subsystems (Patrick, 1992). As system, training is only part of a larger system which can be a company or organization. As defined by Manpower Services Commission (1981), systematic training is "training undertaken on a planned basis as a result of applying a logical series of steps.” In general terms, the steps include: 
- Development of training policy

- Identification of training needs

- Development of training objectives and plans

- Implementation of planned training

- Validation, evaluation, and review of training

The analysis of the development of into its subsystem has given rise to various models, collectively known as Instructional Systems Development models (ISD). These models attempt to analyse the development of training into a series of goals or decisions facing the training manager, although they do not necessarily specify how the goals should be achieved. Among the most recognized ISD models is Patrick's model, which is the adaptation of Eckstrand's training system (see Patrick, 1992), as illustrated in figure 3.

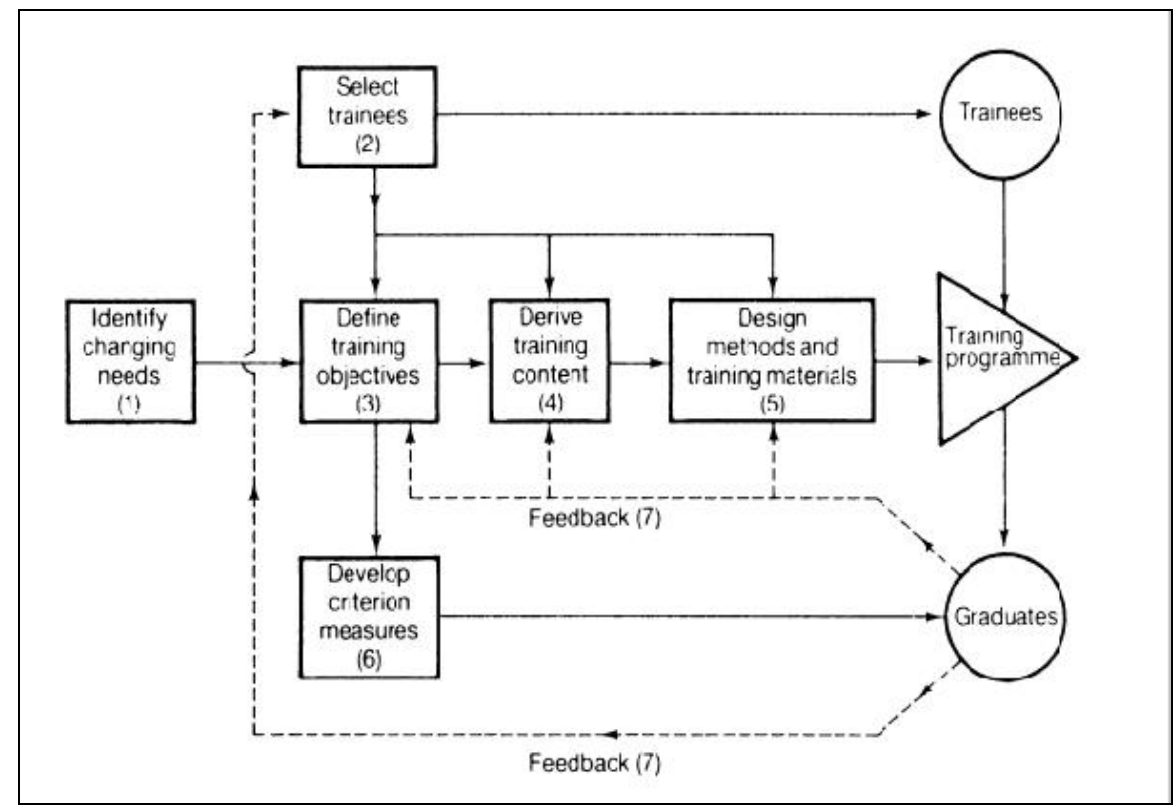

Figure 3: Patrick's ISD model adapted from Eckstrand's training system (source:

Patrick, 1992). 
As the figure shows, the development of training involves six major functions. On top of the list is to identify some actual needs in the organization. This is similar to the second step suggested by Manpower Services Comission (1981). Upon identifying the needs, the goals of the training programmes, which are articulated in detailed behavioural or performance objectives, need to be specified, while selection of trainees is being carried out. Appropriate criterion measures then can be developed on the basis of such goals or objectives, and based on job or task analysis, appropriate training content for the objectives can be derived. Designing methods and training materials is the next step to be taken before finally the training programme can be implemented, and the trainees emerge as graduates of the programme. Evaluation of the training programme by means of its graduates will result in feedback which can be used as basis for any improvement.

In addition to the above model, some other influential ISD models are Inter Services Procedures for Instructional System Development (IPISD), Briggs and Wager's ISD model, and Learning Systems Development (LSD) model, developed by Patrick and his colleagues (Patrick, 1992). In general, all of these models identify generalisable functions in the development of training. The general characteristic of ISD models is that they view the development of training as a system and break it down into subsystems and the functions they perform. In ISD models, the functions involved in training development area prescribed together with the sequence in which they should be carried out. In large-scale organizations, an ISD model will function in facilitating coordination of the training activities. It also provides an evaluation framework in the sense that training can be evaluated by examining the product(s) of training, as well as the process of training development. However, ISD models are not without drawbacks. Patrick (1992) points out two main disadvantages of an ISD approach. Firstly, it arguably presents a too-idealistic perspective and unclear training development activities which are often peculiar to particular training needs. Secondly, they do not provide detailed guidelines of how the training functions should be performed. In other words, they only specify 'what to do' but not 'how to do it'. 


\section{More Recent Training Theories}

Training can generally by categorized into training in the public sector and training in the private sector. The difference between the two lies mainly on their basich philosophy. While training in the private sector focuses more on the jobfocused and technical skills, as well as the high performance of the trainees, which are expected to result in the increase in productivity and profitability, the nature of training in the public sector has changed to be broader in scope in that it has got closer and closer to education. The distinction between training and education has become more and more unclear in recent years.

Kragers (2003) noticed that during the last decade of the twentieth century and the early years of the twenty first century, the tremendous surge of research in training had resulted in more attention given by organizations to employee training. Such attempts in enhancing employee's competencies have provoked the emergence of new approaches and theories in training, although the focus of research remains on mainly on specific methods of training. Among such new theories are Dyadic Protocols, Training Simulators, and Computer-Based Instruction, Team Training, Cross Cultural Training, and Alternative Corporate Model of Training. This emergence of new methods in training has partly been affected by the invention of the new technology in ICT and the broader application of such technology.

When talking about training methods, some taxonomies have been developed by researches to categorized training methods according to their characteristics. One categorization by one of those taxonomies proposed three categories of training methods based on the type and level of trainees, namely: information presentation, information processing, and simulation (Heneman, et al., 1989).

In Information Presentation methods, the design focuses on the presentation of large amount of material to participants in the manner that allows trainee to absorb and memorize non-complex facts. This approach enables independent learning by trainees, where interaction between them is not necessary. Consequently, this method is most useful it aims at knowledge acquisition in which the knowledge is not complex, trainees are self-motivated and capable of learning but there area time or cost limitation (Heneman et al., 1989). 
Information Processing postulates that generation of and discussion on the material to be learned are required to be done by the trainees. In order to do this, they should interact with each other or with the instructor. This method is most appropriate for rapid increases in knowledge, increases in the consistency of performance, and enhancing knowledge already possessed.

As its name implies, Simulation method attempts to imitate the work environment and situations. In this method, interactions of trainees may or may not be required, but the trainees are demanded to have a high level of activity. Included in some methods of simulations are case study, role playing, in-basket exercises, work simulation at mock-up work site (either realistic or simulated), and businessgames (Heneman, et al., 1989). The objectives of simulation method is to develop skills through practice, to change employee attitudes, and to increase diagnostic and problem solving skills.

A question raised in association with training is that are all those theories above completely vacuum or free of any influence of learning theories? A long debate with varied arguments can be presented about it, but when we go through all those training theories carefully, it is not deniable that most of those theories seem to have a base ground in learning theories. Some of them are even just an application of some learning theories. Gagne, Briggs, and Wagger (1992) stated that use of the training (process) is to make effective the process of learning by arranging conditions so that trainee could learn more rapidly and effectively. This confirms how training depends very much on the learning theories.

\section{Conclusions}

The discussions on all of the previously presented theories of learning and theories of training have led to the following conclusions

Theories of learning have long been established with a firm ground philosophically, psychologically, and pedagogically. This strong foundation has made learning theories such a trade mark that looks so firm, stable and reliable. Theories like Behaviorist Approach, Cognitive Learning, and Andragogy are well 
recognized and have become household names for reference when coming to research and study.

Do we have theories of training equally comparable to theories of learning? We have various theories of training, yes, thanks to those researchers who have been particularly committed to the area of human resource development. However, all those theories are actually more practical than theoretical, at least when we consider their philosophical ground. From the philosophical standpoint, it is not deniable that most of the training theories are developed upon the grounds of the learning theories.

As the general conclusion, it seems appropriate, therefore, to say that there are indeed some theories of training that are applicable for individual and organizational training. However, such theories are obviously not equally comparable to the theories of learning, which in many cases are used as their basis. It still requires more efforts and harder works of training researchers to prove that the training theories can stand equally in the same level as the learning theories.

\section{Refferences}

Al-Khayyat, R.M. and Elgamal, M.A. (1997), 'A macro model of training and development: validation'. Journal of European Industrial Training, 21/3.

Gagne, R. M, Briggs, L. J and Wager, W. W,(1992) Principles of Instructional Design, 4 th ed. Harwart trace Jovanovich college publisher

Garavan, T.N. (1997), 'Training, development, education and learning: different or the same?' Journal of European Industrial Training, 21/2.

Goldstein, I.L. (1986), Training in Organizations: Needs Assessment, Development, and Evaluation, Monterey, California: Brooks/Cole.

Hackett, P. (1997), Introduction to Training, London: IPD.

Harrison, R (1997), Employee Development, Wiltshire: The Cromwell Press.

Heneman, H.G., III, Schwab, D.P., Fossum, J.A., \& Dyer, L.D. (1989). Personnel Human Rresource Management.Boston: Irwin 
Hinrichs, J.R. (1976), 'Personal Training'. In Dunnette, M.D.(Ed), Handbook of Industrial and Organizational Psychology, Chicago: Rand McNally.

Knowles, M.S., Holton III, E.F., and Swanson, R.A. (1998), The Adult Learner, Houston, Texas: Gulf Publishing.

Krager, K. (2003), Perspectives on Training and Development, http://www.owlnet.rice edu/ ajv2/courses/12a psyc630001/Kraiger\%20\%282003\%29.pdf

Manpower Services Commission (1981), Glossary of Training Terms, London: HSMO.

Mullins, L.J., (1999), Management and Organisational Behaviour, London: Prentice Hall.

Patrick, J. (1992), Training: Research and Practice, London: Academic Press.

Sloman, M. (1994), A Handbook for Training Strategy, Aldershot, Hampshire: Gower.

Stammers, R., and Patrick, J. (1975), Psychology of Training, London: Methuen.

Stewart, J. (1999), Employee Development Practice, Harlow, Essex: Pearson Education 